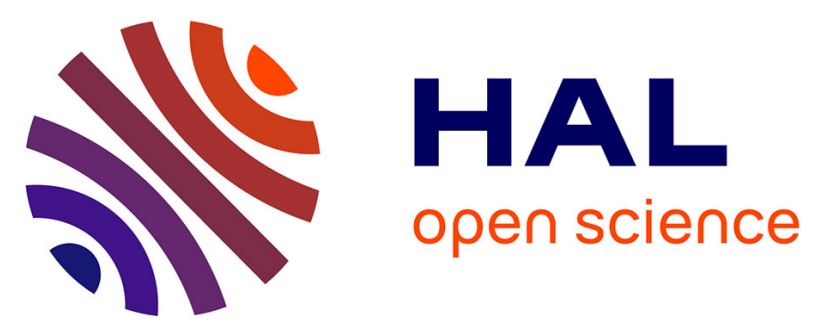

\title{
Construction and test of a 1x1 m2 Micromegas chamber for sampling hadron calorimetry at future lepton colliders
}

C. Adloff, I. Blaha, M. Chefdeville, A. Dalmaz, C. Drancourt, A. Espargilière, R. Gaglione, N. Geffroy, D. Girard, J. Jacquemier, et al.

\section{To cite this version:}

C. Adloff, I. Blaha, M. Chefdeville, A. Dalmaz, C. Drancourt, et al.. Construction and test of a 1x1 m2 Micromegas chamber for sampling hadron calorimetry at future lepton colliders. Nuclear Instruments and Methods in Physics Research Section A: Accelerators, Spectrometers, Detectors and Associated Equipment, 2013, 729, pp.90-101. 10.1016/j.nima.2013.06.081 . in2p3-00909211

\section{HAL Id: in2p3-00909211 https://hal.in2p3.fr/in2p3-00909211}

Submitted on 26 Nov 2013

HAL is a multi-disciplinary open access archive for the deposit and dissemination of scientific research documents, whether they are published or not. The documents may come from teaching and research institutions in France or abroad, or from public or private research centers.
L'archive ouverte pluridisciplinaire HAL, est destinée au dépôt et à la diffusion de documents scientifiques de niveau recherche, publiés ou non, émanant des établissements d'enseignement et de recherche français ou étrangers, des laboratoires publics ou privés. 


\title{
Construction and test of a $1 \times 1 \mathrm{~m}^{2}$ Micromegas chamber for sampling hadron calorimetry at future lepton colliders
}

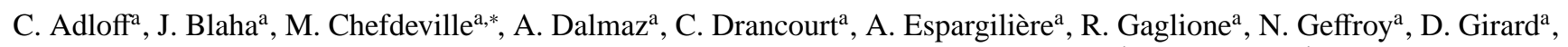 \\ J. Jacquemier ${ }^{\mathrm{a}}$, Y. Karyotakis ${ }^{\mathrm{a}}$, I. Koletsou ${ }^{\mathrm{a}}$, F. Peltier ${ }^{\mathrm{a}}$, J. Samarati ${ }^{\mathrm{a}}$, S. Tsigaridas ${ }^{\mathrm{b}}$, G. Tsipolitis ${ }^{\mathrm{b}}$, G. Vouters ${ }^{\mathrm{a}}$ \\ ${ }^{a}$ LAPP, Laboratoire d'Annecy-le-Vieux de Physique des Particules, Université de Savoie, CNRS/IN2P3, 9 Chemin de Bellevue - BP 110, 74941 Annecy-le-Vieux \\ Cedex, France \\ ${ }^{b}$ NTUA, National Technical University of Athens, Zografou Campus, GR-157, 80 Zografou, Greece
}

\begin{abstract}
Sampling calorimeters can be finely segmented and used to detect showers with high spatial resolution. This imaging power can be exploited at future linear collider experiments where the measurement of jet energy by a Particle Flow method requires optimal use of tracking and calorimeter information. Gaseous detectors can achieve high granularity and a hadron sampling calorimeter using Micromegas chambers as active elements is considered in this paper. Compared to traditional detectors using wires or resistive plates, Micromegas is free of space charge effects and could therefore show superior calorimetric performance. To test this concept, a prototype of $1 \times 1 \mathrm{~m}^{2}$ equipped with 9216 readout pads of $1 \times 1 \mathrm{~cm}^{2}$ has been built. Its technical and basic operational characteristics are reported.
\end{abstract}

Keywords: Large area Micromegas, Micro Pattern Gaseous Detectors, Digital hadron calorimetry, Future linear colliders

\section{Introduction}

\subsection{Particle Flow calorimetry}

The detailed study of electroweak symmetry breaking and of

4 the properties of the Higgs boson within and beyond the Stan- ${ }_{31}$ dard Model (SM) are some of the physics goals motivating the ${ }_{32}$ construction of a linear electron positron collider (ILC or CLIC ${ }_{33}$ $[1,2])$. This physics case is now enhanced with the discov- ${ }_{34}$ ery at LHC of a Higgs-like new particle [3, 4]. Many interest- ${ }_{35}$ ing physics channels at a linear collider will be reconstructed ${ }_{36}$ in multi-jet final states, often accompanied by charged leptons ${ }_{37}$ and missing transverse energy associated with neutrinos or pos- ${ }_{38}$ sibly the lightest super-symmetric particles. The reconstruction ${ }_{39}$ of the invariant masses of two or more jets will be important ${ }_{40}$ for event reconstruction and event identification. The dijet mass ${ }_{41}$ resolution should be good enough to identify $Z$ and $W$ bosons in ${ }_{42}$ their hadronic final states with an accuracy comparable to their ${ }_{43}$ natural decay width. This requires an excellent jet energy res- ${ }_{44}$ olution of 3-4\% over an energy range extending up to $1.5 \mathrm{TeV}_{45}$ for a $3 \mathrm{TeV}$ collider.

Two techniques are studied by the DREAM [5] and CAL- ${ }_{47}$ ICE [6] collaborations to meet this goal. The first one, called Dual Readout, is a compensation technique that uses cherenkov ${ }_{49}$ and scintillation light produced in hadron showers to correct ${ }_{50}$ for fluctuations of the electromagnetic fraction which other- ${ }_{51}$ wise dominate the jet energy resolution [7]. The Particle Flow ${ }_{52}$ technique relies on highly segmented calorimeters and a precise ${ }_{53}$ tracker to separate the jet's charged and neutral components [8]. ${ }_{54}$

\footnotetext{
${ }^{*}$ Corresponding author

Email address: chefdevi@lapp.in2p3.fr (M. Chefdeville)
}

28 The use of the tracking information reduces the dependence on ${ }_{29}$ hadronic calorimetry and results in the required excellent jet energy and di-jet mass resolution [9].

\subsection{Semi-digital hadron calorimetry}

Two hadron calorimeters using steel or tungsten absorbers are developed by the the CALICE collaboration. The first is instrumented with $3 \times 3 \mathrm{~cm}^{2}$ scintillating tiles read out by SiPM and 12 bit ADCs [10]. The second uses gaseous detectors with higher segmentation $\left(1 \times 1 \mathrm{~cm}^{2}\right)$ and simpler readout $(1$ bit or 2 bit $[11,12])$. The first favours single hadron energy resolution (higher sampling fraction, analogue readout) while the second targets a high shower separation capability (smaller cells) probably at the expense of resolution (digital readout).

A digital hadron calorimeter (1 bit, DHCAL) is expected to have two regimes of operation. A low energy linear regime where the response to the electromagnetic and hadronic shower parts, taken separately, is constant. In this regime, Landau fluctuations are suppressed resulting in improved resolution with respect to a perfect analogue readout. A higher energy saturated regime where the energy information is lost due to undercounting and the resolution degrades with increasing hadron energy $[13,14]$. The energy frontier between the two regimes depends mainly on the cell size and absorber material. In an SiDlike HCAL geometry [15] $\left(1 \times 1 \mathrm{~cm}^{2}\right.$ pads, steel absorbers), Monte Carlo studies indicate a frontier between $20-30 \mathrm{GeV}$.

The electromagnetic part of hadron showers results in dense energy deposits and is responsible for the saturation of a DHCAL. A way to account for these deposits in the energy reconstruction is to use additional readout thresholds (2 bit, semidigital HCAL or SDHCAL). With the right threshold settings 
and energy reconstruction algorithm, it should be possible to improve the energy resolution beyond the saturated regime.

\subsection{The Micromegas detector and calorimeter project}

Micromegas is a Micro Pattern Gas Detector (MPGD) that uses a thin mesh to separate the gas volume into two regions [16]. A low field region where primary electrons are released from the atoms and a high field region where they are drifted to and multiply by avalanche. Thanks to a fast collection of the avalanche ions, Micromegas is free of space charge effects up to very high particle rates and therefore well suited for tracking in high rate environments. This property also makes this detector very appealing for calorimetry because the sum of the anode signals is proportional to the energy deposited in the drift region. This is an improvement with respect to wire chamber based gaseous calorimeters which suffered from intrinsic signal saturation from the ion space charge around the wires [17]. In addition, ageing effects in Micromegas are minimal because it operates in simple gas mixtures (e.g. $\mathrm{Ar} / \mathrm{CO}_{2}$ ) and at relatively low electric fields $(\sim 40 \mathrm{kV} / \mathrm{cm}$ with a gap of $128 \mu \mathrm{m})$.

The Micromegas calorimeter project was initiated in 2006. The first step of the project was the characterisation of small prototypes equipped with off-detector electronics. Based on the successful results [18], the project moved on to the next phase by integration of the electronics on the detector printed circuit ${ }_{110}$ board (PCB) and by scaling up of the detector dimensions.

\section{Description of the $1 \times 1 \mathrm{~m}^{2}$ Micromegas prototype}

\subsection{Active sensor units}

An Active Sensor Unit (ASU) is a $32.4 \times 48.4 \mathrm{~cm}^{2} \mathrm{PCB}^{11}$ (8 layers, $1.2 \mathrm{~mm}$ thin) segmented into 1536 anode pads of ${ }^{117}$ $1 \times 1 \mathrm{~cm}^{2}$ arranged in 32 rows and 48 columns. It is equipped ${ }^{118}$ with a Micromegas mesh and 24 front-end chips. The mesh is ${ }^{119}$ laminated on the PCB pad plane according to the Bulk process ${ }^{120}$ [19] and held by small equally spaced pillars and $2 \mathrm{~mm}$ wide ${ }^{121}$ strips on the four ASU edges. Packaged chips are soldered to ${ }^{122}$ the opposite PCB side, together with gas discharge protection ${ }^{123}$ diodes, polarisation resistors, high voltage decoupling capaci- ${ }^{124}$ tors and flat connectors.

The ASU chips are read out with 2 Detector Interface boards ${ }^{126}$ (DIF, inter-DIF) which also distribute voltage to the front-end ${ }^{127}$ electronics and to the Micromegas mesh. ASU and inter-DIF ${ }^{128}$ are connected with flat cables to minimise the detector thick- ${ }^{129}$ ness and to allow for some mechanical flexibility between the $2^{130}$ boards. Thanks to flat connectors on both sides of the ASU, sev- ${ }^{13}$ eral ASUs can be read out in a row (Figure 1). This is essential ${ }^{132}$ for constructing large chambers as several ASUs can be chained ${ }^{133}$ and read out with only one pair of DIF/inter-DIF boards.

\subsection{Front-end electronics}

The ILC beam will be pulsed and composed of $1 \mathrm{~ms}$ long $_{138}$ bunch trains separated by $199 \mathrm{~ms}$. During a train, bunches col- ${ }_{139}$ lide every $340 \mathrm{~ns}$ and detector signals are digitised and associ- ${ }_{140}$ ated to the time of a bunch. Between trains, all information is ${ }_{141}$ read out from the memory to the back-end electronics and the

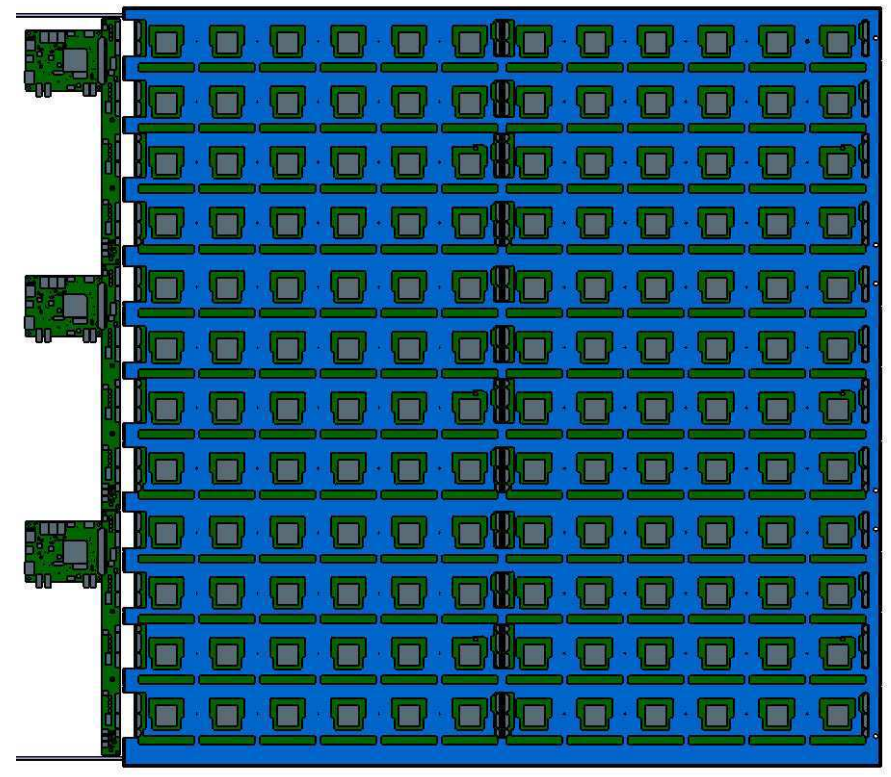

Figure 1: Chip side of the $1 \times 1 \mathrm{~m}^{2}$ prototype showing the readout boards on the left side (three pairs of DIF and inter-DIF), six ASUs glued to a rigid mask (in blue) and the gas inlet and outlet at the top and bottom left corners.

front-end circuits are turned off to reduce the heat dissipation inside the calorimeters. Key electronics features are thus selftriggering with memory, time-stamping and power-pulsing.

A front-end chip optimised for the detection of Micromegas signals has been developed [20]. It is called MICROROC (Micromegas Readout Chip) and belongs to a generation of chips optimised for calorimetry at a future linear collider [21]. The MICROROC is a 64 channel chip, with three readout thresholds and a power-pulsing capability to reduce its consumption from a nominal value of $3.7 \mathrm{~mW}$ at $3.5 \mathrm{~V}$ per channel to $20 \mu \mathrm{W}$ (assuming a duty cycle of $0.5 \%$ ). Each channel input is protected against gas discharges by a diode network followed by a charge preamplifier and two shapers of low and high gain and tunable peaking time (75-200 ns). The shaper outputs are connected to three discriminators. When a signal crosses the low threshold, the content of the 64 channel matrix is written to memory with a clock time (so-called event). A total of 127 events can be recorded before filling completely the chip memory. The latter is read out either when it is full (self-trigger mode) or upon the arrival at the chip of an external trigger signal (trigger mode).

The high gain shaper is connected to the low and medium threshold discriminators and has a dynamic range of $200 \mathrm{fC}$. The low gain shaper has a linear response up to $500 \mathrm{fC}$ and is connected to the high threshold discriminator. The 3 thresholds are set by 10 bit DACs common to the 64 channels. Each channel features a 4 bit DAC that can be used to shift the pedestal voltage with respect to the common thresholds and minimise their dispersions. A detailed characterisation of the detector can be performed with a calibration test input and a multiplexed shaper output (analogue readout). The calibration of the electronics is presented in section 3.1 and the analogue readout of the shaper signals is illustrated in section 4.7 . 


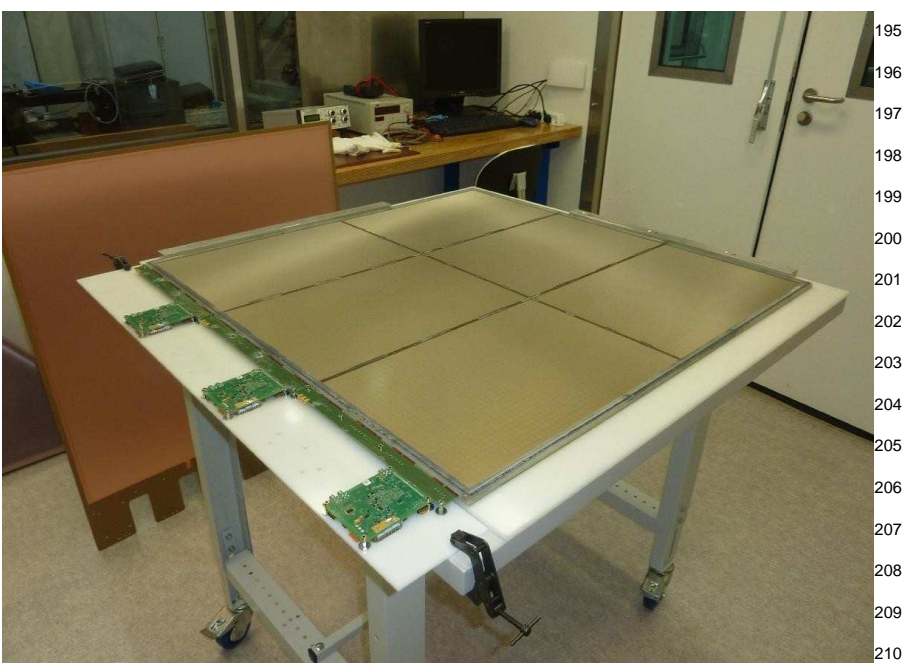

Figure 2: The $1 \times 1 \mathrm{~m}^{2}$ prototype during assembly showing the mesh side of the six ASUs, the readout boards and the cathode cover.

The total chamber thickness is $9.2 \mathrm{~mm}$ which includes ${ }^{213}$ $2.7 \mathrm{~mm}$ for the cathode cover, $3 \mathrm{~mm}$ of drift gap and $3.5 \mathrm{~mm}_{215}$ for PCB, ASICs and mask. With this mechanical design, the ${ }_{216}$ fraction of non-instrumented area is $1.5 \%$ of the total area defined by the six ASUs. Dead zones are mainly caused by the ${ }_{218}$ $1 \mathrm{~mm}$ gap between ASUs and the $2 \mathrm{~mm}$ wide inactive photore- ${ }_{219}$ sist strips that support the mesh on the four ASU sides.

The mechanical constraints to build an ILC hadron calorime-176 ter are stringent. The calorimeter will be located inside the ${ }^{177}$ olenoid magnet which limits its total depth to preserve cost; 178 envisaged space between absorbers is $8 \mathrm{~mm}$. The font-end 17 will be integrated in the active layers to minimise dead 180 the module edges. Another challenge is the fabrication of large ${ }^{182}$ area active layers (up to $1 \times 3 \mathrm{~m}^{2}$ in the SiD design).

Modular and scalable to larger area, the $1 \times 1 \mathrm{~m}^{2}$ Micromegas $^{185}$ prototype consists of six ASUs glued on a rigid mask and placed ${ }^{186}$ single gas volume (Figure 1 and 2). Small spacers are incover, defining prec tic frames are closing the chamber sides, leaving openings for ${ }^{190}$ two gas pipes and flat cables for electronics connections. After assembly, the chamber is equipped with readout boards (three ${ }^{191}$ pairs of DIF/inter-DIF) and a patch panel for voltage distribu- ${ }^{192}$ tion.

\section{.}

\section{-}

\section{(a)} dominate the response uniformity of this detector [18].

The low threshold discriminator triggers the writing to memory of the 64 channel content. It is connected to the output of the high gain shaper and therefore only the noise of this shaper is relevant for our purposes. Calculating the noise as $\sigma$ divided by the gain, an average noise of $0.25 \mathrm{fC}$ is found with variations of $0.03 \mathrm{fC}$ RMS over all channels (Figure 5). This is quite small compared to a typical minimum ionising particle (MIP) signal of 1-20 fC and close to what was measured before soldering the chips to the PCBs (in the latter case, a capacitor equal to the detector pad to ground capacitance was connected to the chip inputs). In conclusion, neither the design of the PCB nor the mesh lamination increase the noise level at the channel inputs.

\subsubsection{Thresholds and pedestals}

The three discriminator DACs of a MICROROC are common to the 64 channels. The lowest possible threshold is therefore determined by the channel with the highest pedestal, for instance $5 \sigma$ above this pedestal, channels with lower pedestals experiencing larger thresholds. To minimise the threshold spread, each channel is equipped with a pedestal DAC. The latter controls the pedestal voltage and can be used to correct the individual thresholds by a few fC. A method to align the pedestals is to set the pedestal DACs to obtain a uniform noise rate over all the channels. With this method, the spread of thresholds is reduced by a factor of 2-3 (Figure 6). The improvement of the detector response is significant when operating at a moderate gas gain $(\leq 1000)$.

In a semi-digital calorimeter, the values of the medium and high thresholds should be optimised for best energy resolution over the relevant energy range. During the test beam period reported in section 4 , several settings were tried. 


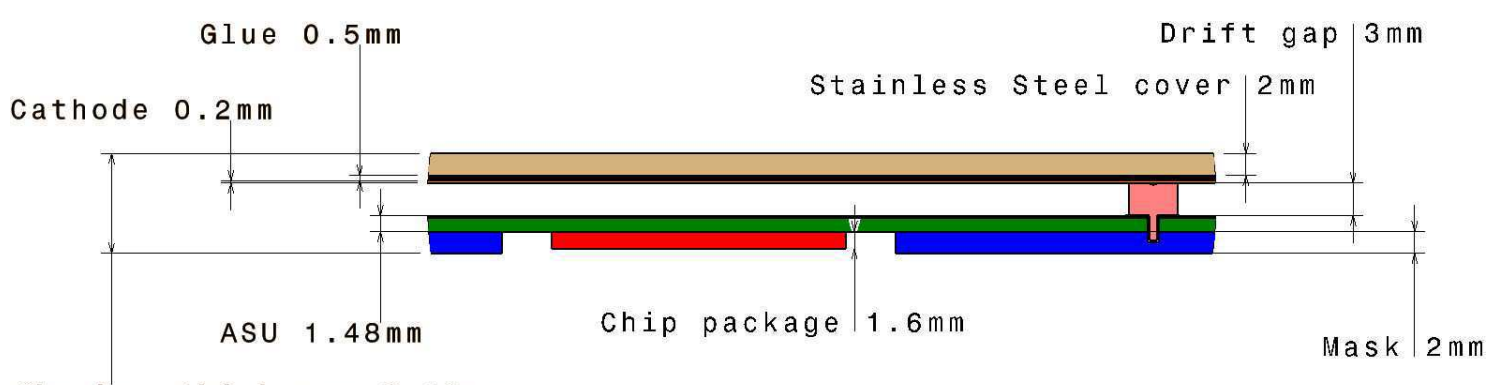

Chamber thickness $9.18 \mathrm{~mm}$

Figure 3: Cross-section of the $1 \times 1 \mathrm{~m}^{2}$ prototype close to a junction between two ASUs. The spacer on the right side defines the $3 \mathrm{~mm}$ drift gap.
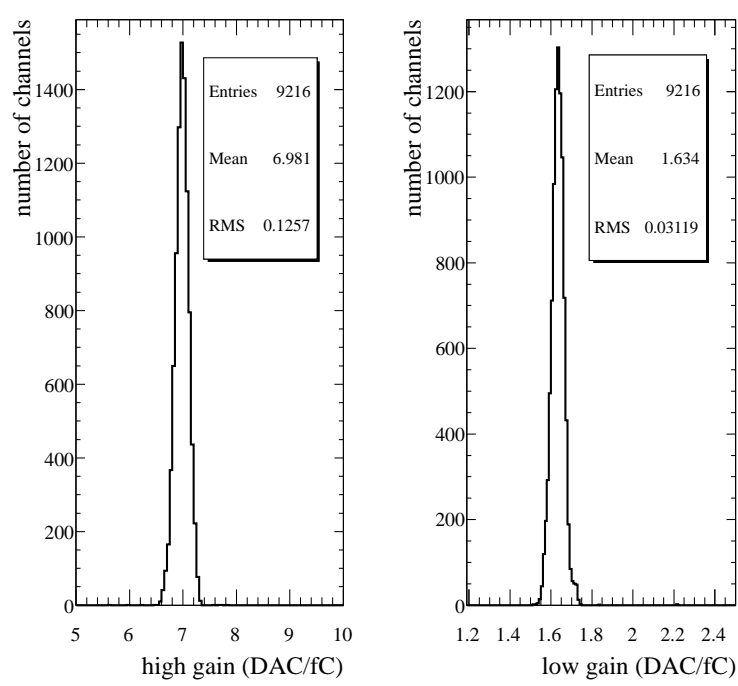

Figure 4: Shaper gains measured on all channels of the $1 \times 1 \mathrm{~m}^{2}$ prototype.

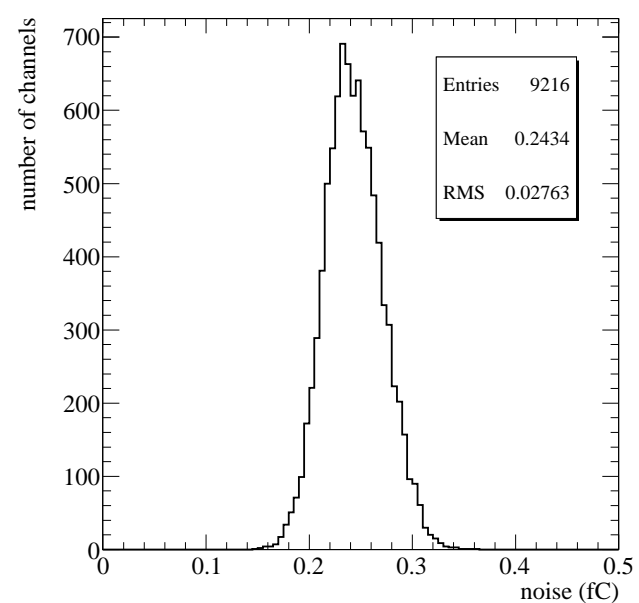

Figure 5: Noise at the output of the high gain shaper for all channels of the ${ }^{24}$ $1 \times 1 \mathrm{~m}^{2}$ prototype
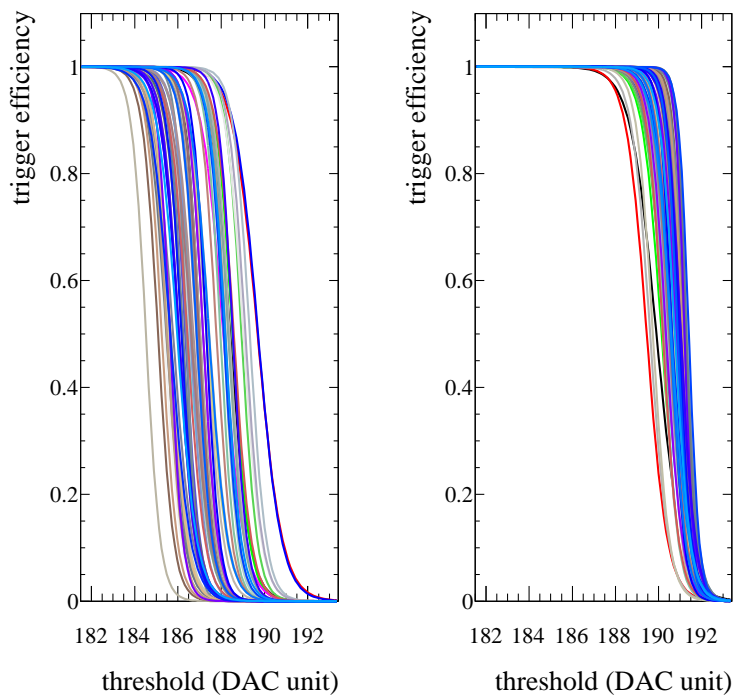

Figure 6: Channel pedestals of one chip measured during a threshold scan before (left) and after (right) alignment of the noise rate.

\section{2. $X$-ray tests}

Counting experiments are performed with an ${ }^{55} \mathrm{Fe} 5.9 \mathrm{keV}$ X-ray source to characterise all ASUs before they are sealed in the $1 \times 1 \mathrm{~m}^{2}$ prototype. A dedicated gaseous chamber with $14 \mathrm{~mm}$ drift gap and perforated drift cover has been constructed to measure the response of any of the 1536 ASU channels to true Micromegas signals.

In a non-flammable mixture of $\mathrm{Ar} / \mathrm{CF}_{4} / \mathrm{iC}_{4} \mathrm{H}_{10} 95 / 3 / 2,{ }^{55} \mathrm{Fe}$ quanta can convert in the gas mainly by a photoelectric effect on an argon atom. This interaction results on average in 115 or 230 primary electrons depending on the atom relaxation process: fluorescence (escape peak) or Auger cascade (photopeak) [22]. After drifting, almost all primary electrons are multiplied in the amplification gap [18]. If above threshold, pad signals are recorded as hits in the chip memory. An external trigger signal is used to read out the memory every $1 \mathrm{~s}$. The counting rate was measured for various sets of experimental parameters (thresholds, mesh voltage and source position). Each run lasted $60 \mathrm{~s}$ and the drift field was set to the local maximum of the drift velocity. Results are presented and discussed below. 


\subsubsection{Threshold scans}

The gas gain is deduced from measurements of the counting rate $R$ versus threshold $t$ at various mesh voltages. Low threshold scans were performed at voltages between 300 and $350 \mathrm{~V}$. With the field settings used, the expected average spread of a point-like cloud of electrons (from a photoelectric conversion) at the mesh is $\sim 230 \mu \mathrm{m}$ in the direction transverse to the field and $\sim 2 \mathrm{~ns}$ in time [23]. With the source collimated to the centre of a pad, most primary electrons are collected on one pad. For simplicity all other pads were electronically disabled. The results are shown in Figure 7. Each $R(t)$ trend is well described by the sum of two sigmoid functions accounting for the photopeak (peak 1) and the escape peak (peak2):

$$
R(t)=\frac{R_{1}}{1+\exp \left(\frac{t-t_{1}}{\Delta t_{1}}\right)}+\frac{R_{2}}{1+\exp \left(\frac{t-t_{2}}{\Delta t_{2}}\right)}
$$

where the parameters $\left(R_{1}, R_{2}\right)$ are the rates at zero threshold, $\left(t_{1}, t_{2}\right)$ the inflexion thresholds at the peak maxima and $\left(\Delta t_{1}\right.$, $\left.\Delta t_{2}\right)$ are proportional to the peak widths. In order to reduce the number of parameters fitted to the data points, the following approximations between the two peaks are used:

$$
\frac{R_{1}}{R_{2}}=\frac{1-f}{f}=\frac{85}{15}
$$

where $f$ is the fluorescence yield of an excited argon atom [24]. Noting $E_{1}$ and $E_{2}$ the mean energy of the photopeak and escape peak:

$$
\frac{t_{1}}{t_{2}}=\frac{E_{1}}{E_{2}}=2
$$

$$
\frac{\Delta t_{1}}{\Delta t_{2}}=\sqrt{\frac{E_{1}}{E_{2}}}=\sqrt{2}
$$

and Equation 1 becomes:

$$
R(t)=R_{1}\left[\frac{0.85}{1+\exp \left(\frac{t-t_{1}}{\Delta t_{1}}\right)}+\frac{0.15}{1+\exp \left(\frac{t-0.5 t_{1}}{\Delta t_{1} / \sqrt{2}}\right)}\right]
$$

After the fit, all hit rates converge to roughly $8 \mathrm{~Hz}$ at zero threshold. The measured charge at the various inflexion points $\left(t_{1}\right)$ is used to calculate the gas gain, assuming an average of 230 primary electrons for photopeak events. The gain exhibits the usual exponential dependence on the mesh voltage (Figure 8) with a slope of $0.036 / \mathrm{V}$ typical of argon-based gas mixtures [25]. At $350 \mathrm{~V}$, a scan of the high threshold was performed too. The resulting $R(t)$ trend is shown in Figure 9 together with the low threshold trend. The two threshold scans give gas gain values of 323 and 300 respectively. The agreement is reasonable and the $4 \%$ difference can probably be explained by systematic errors during the calibration.

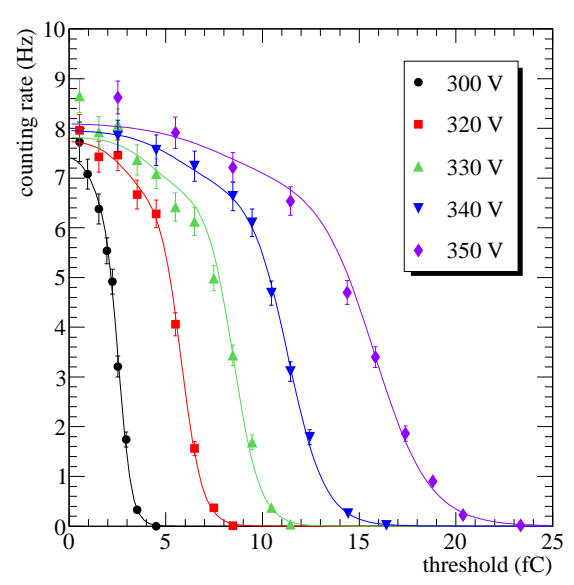

Figure 7: ${ }^{55} \mathrm{Fe}$ quanta counting rate of one channel versus low threshold at various mesh voltages.

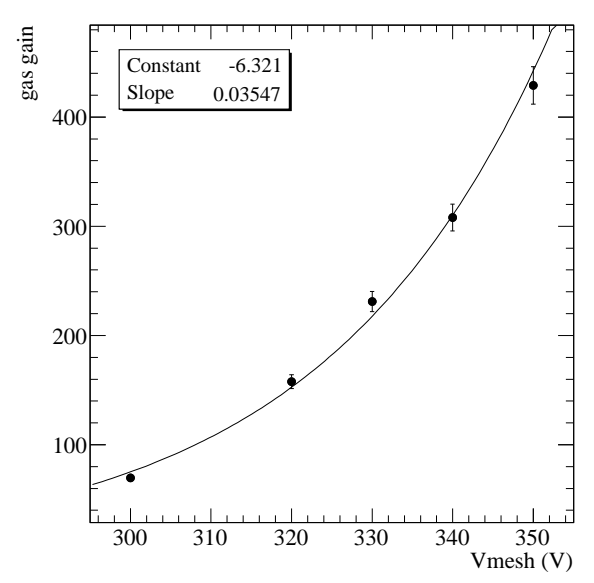

Figure 8: Gas gain versus mesh voltage deduced from the threshold scans. Data points are described by the formula $G=\exp \left(A+B V_{\text {mesh }}\right)$ with $A \sim-6.32$ and $B \sim 0.036 / \mathrm{V}$.

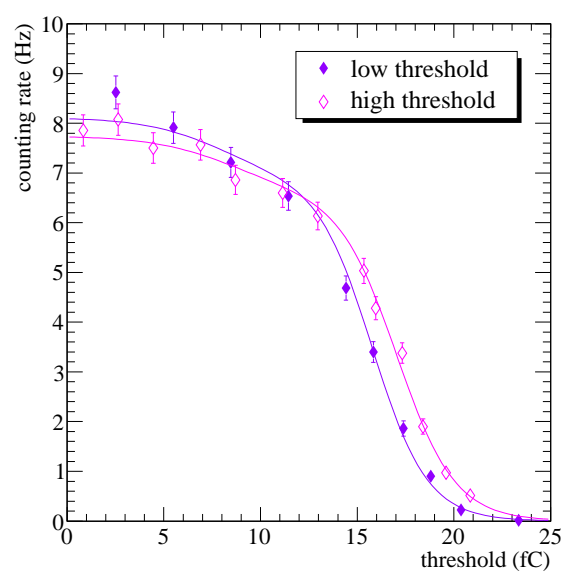

Figure 9: ${ }^{55} \mathrm{Fe}$ quanta counting rate of one channel versus low and high thresholds at a mesh voltage of $350 \mathrm{~V}$. 


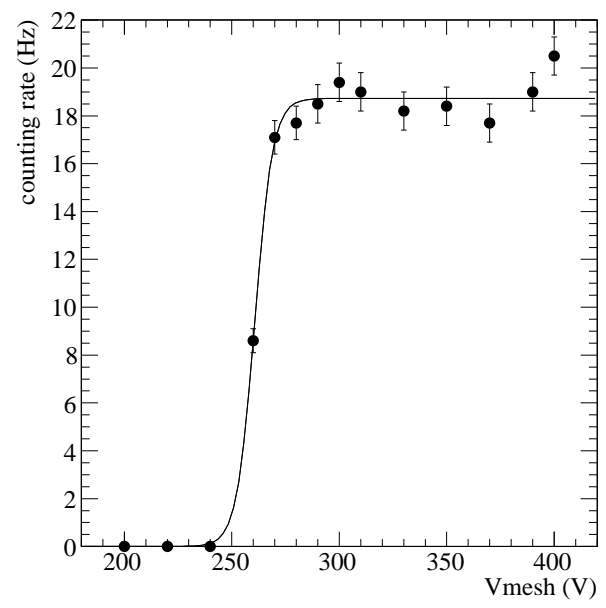

Figure 10: ${ }^{55} \mathrm{Fe}$ quanta counting rate of one channel versus mesh voltage.

\subsubsection{Mesh voltage scan}

The smallest detectable charge is deduced from a measurement of the counting rate versus gas gain. In this study, the source is collimated to the centre of one pad while the other pads are disabled. The low threshold of the tested pad is set by iteratively decreasing the chip discriminator DAC until the count rate becomes dominated by noise. The final DAC value is set one unit above this steep transition and this configuration is defined as the configuration of lowest workable threshold. The counting rate is then measured at various mesh voltages (200$400 \mathrm{~V}$ ) in this threshold configuration. As shown in Figure 10, it increases with voltage as the charge spectrum shifts above threshold. The trend can be described by an sigmoid function with an inflexion point at $260 \mathrm{~V}$. At this voltage, the rate is by definition half of its maximum value which implies that the threshold is equal to the average pad charge. The smallest detectable charge is then:

$$
Q=q_{\mathrm{e}} N G=1.6 \cdot 10^{-4} \cdot 212 \cdot 20 \approx 0.7 \mathrm{fC}
$$

where $N$ is averaged over the ${ }^{55} \mathrm{Fe}$ spectrum (i.e. $0.85 \cdot 230+0.15 \cdot 115)$. Previous measurements showed that a MIP efficiency larger than $95 \%$ is achieved when the most probable value of the charge is roughly three times the threshold [18]. Assuming a most probable number of primary electrons from MIPs of 14 [18], this condition should be met at ${ }_{326}$ a moderate gas gain around 1000 .

\subsubsection{Position scan}

The uniformity of the gas gain and of the low threshold can $_{32}$ be verified by measuring the $\mathrm{X}$-ray counting rate on various po- 330 sitions for all six ASUs. For a given ASU, the position scan sc $_{331}$ is performed on six different positions. At each position the ${ }_{332}$ source is collimated onto a region of $2 \times 2$ pads centred in between four chips (Figure 11). In this way, all 24 ASU chips are 334 involved in the counting experiment. For this study, all channels ${ }_{335}$ are enabled and their thresholds are equalised according to the ${ }_{336}$ procedure explained in section 3.1.3. The mesh voltage is set set $_{33}$

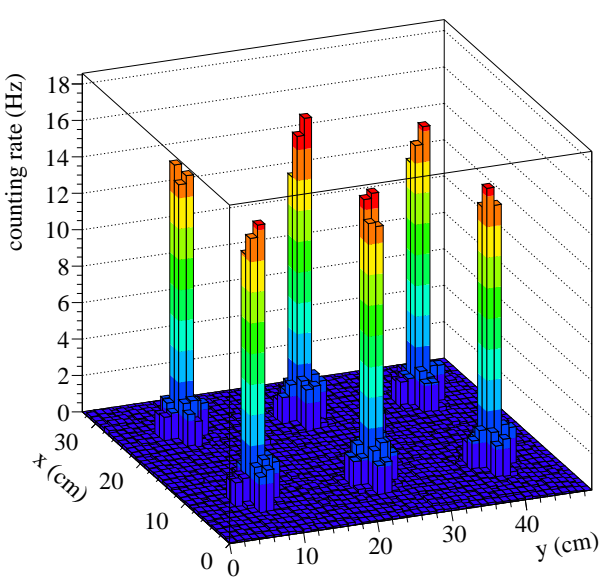

Figure 11: Two-dimensional hit distribution obtained with an ${ }^{55} \mathrm{Fe}$ source successively placed at six positions over the ASU test chamber.

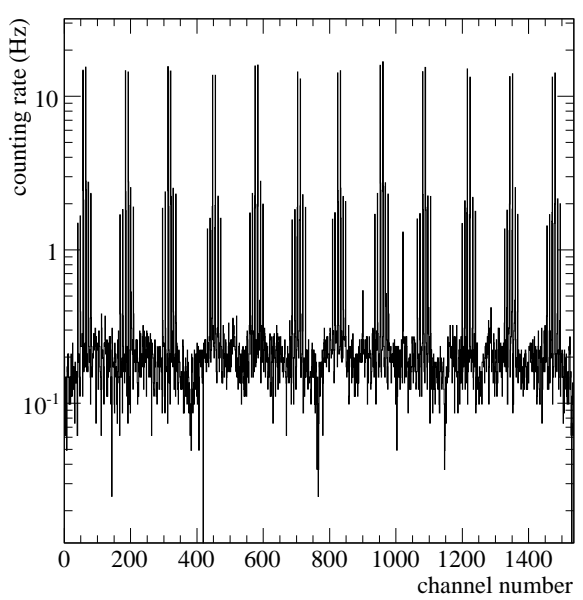

Figure 12: Channel occupancy obtained with an ${ }^{55} \mathrm{Fe}$ source successively placed at six positions over the ASU test chamber.

to $320 \mathrm{~V}$ at which an average ${ }^{55} \mathrm{Fe}$ signal of $\sim 5 \mathrm{fC}$ is expected. Given the collimation of the source and the transverse electron diffusion in the gas, the count rate is now calculated over $8 \times 8$ pad regions.

Position scans have been performed for six ASUs before assembly in the $1 \times 1 \mathrm{~m}^{2}$ prototype. As illustrated in Figure 12, the response of the channels to the source quanta seems uniform. A flat noise-free background from cosmic particles can be seen when plotting the channel occupancy in a logarithmic scale. The results are summarised in Table 1. For each ASU, small variations of the counting rate are observed (the statistical error is $0.4 \mathrm{~Hz}$ ). ASU to ASU variations of the mean rate are a few Hz. They could be caused by change of atmospheric conditions from one test to the other. 


\begin{tabular}{c|ccccccc} 
ASU number & 1 & 2 & 3 & 4 & 5 & 6 & ${ }^{382}$ \\
\hline \hline Mean rate (Hz) & 86.2 & 85.2 & 87.0 & 79.3 & 84.2 & 84.3 & ${ }^{383}$ \\
RMS (Hz) & 2.0 & 1.7 & 1.1 & 1.6 & 2.2 & 2.5 & \\
385 \\
386
\end{tabular}

Table 1: ${ }^{55} \mathrm{Fe}$ quanta counting rates and their variations measured on six ASUs ${ }^{387}$ (six measurements per ASU). The statistical error on the mean is $0.4 \mathrm{~Hz}$.

\subsubsection{Conclusion of the ASU tests}

The manufacturing technique and the calibration procedure ${ }^{392}$ allow to achieve very low detection threshold, negligible noise and good response uniformity in a reproducible way. After careful characterisation of six ASUs, the first $1 \times 1 \mathrm{~m}^{2} \mathrm{Mi}^{-394}$ cromegas prototype with MICROROC readout was constructed in May 2011 and subsequently tested in beam in July 2011. The ${ }^{395}$ results of the test beam are presented in the next section.

\section{Functional tests of the prototype in particle beams}

The goal of the test beam is to validate the mechanical design ${ }_{40}^{400}$ of the $1 \times 1 \mathrm{~m}^{2}$ prototype, to measure its response to MIPs and ${ }_{402}$ to test its main functionalities. The test set-up consists of the ${ }_{403}$ $1 \times 1 \mathrm{~m}^{2}$ prototype and a telescope of small Micromegas cham- ${ }_{404}$ bers and three scintillating paddles of $6 \times 16 \mathrm{~cm}^{2}$ read out by ${ }_{405}$ photomultiplier tubes (PMT) (a detailed description of the tele- ${ }_{406}$ scope is given in [18]). This set-up was installed at the $\mathrm{CERN}_{407}$ SPS facility in the $\mathrm{H} 4$ beam line and exposed to $150 \mathrm{GeV} / \mathrm{c}_{408}$ muons and pions. All chambers were flushed with a gas mix- ${ }_{409}$ ture of $\mathrm{Ar} / \mathrm{CF}_{4} / \mathrm{iC}_{4} \mathrm{H}_{10} 95 / 3 / 2$. The Micromegas pad planes are ${ }_{410}$ vertical and perpendicular to the beam axis. During the pion ${ }_{411}$ runs, a $20 \mathrm{~cm}$ long block of iron $\left(10 \times 10 \mathrm{~cm}^{2}\right.$ cross-section) was $_{412}$ placed between the telescope and the prototype to study its be- ${ }_{413}$ haviour in hadron showers. The trigger is generated by the time ${ }_{414}$ coincidence of the three PMT signals. It is delayed by $1.5 \mu \mathrm{s}_{415}$ before reaching the detectors in order to accommodate for the peaking time of the prototype and telescope electronics (200 ns and $1.2 \mu$ s respectively) and also to check the behaviour of the ${ }^{416}$ prototype after the passage of the beam particles. To account ${ }_{417}$ for the dead time of the telescope and prototype, a gate signal ${ }_{418}$ enters the coincidence such that any PMT signal arriving dur- ${ }_{419}$ ing the readout of the detectors is vetoed. The dead time of the ${ }_{420}$ whole set-up is dominated by the telescope and is $\sim 10 \mathrm{~ms}$.

\subsection{Externally triggered operation}

The contributions from beam muons, cosmics particles and ${ }_{224}$ electronic noise to the $1 \times 1 \mathrm{~m}^{2}$ prototype data are studied $\mathrm{in}_{425}$ trigger mode. To this end, a low intensity muon beam of $250 \mathrm{~Hz}_{426}$ collimated to roughly the size of the scintillators $\left(\sim 100 \mathrm{~cm}^{2}\right)$ is ${ }_{427}$ used. The mesh voltage is set to $370 \mathrm{~V}$ at which a MIP effi- 428 ciency larger than $95 \%$ is reached (cf. section 4.4). The thresholds are equalised according to the procedure previously de-429 scribed, resulting in a number of disabled channels of $10 . \quad 430$

In Figure 13, the counting rate of roughly one fifth of the 431 prototype channels is shown. Broad peaks correspond to beam ${ }_{432}$ muons and reach a maximum counting rate of $\sim 3.8 \mathrm{~Hz} /$ channel $_{433}$ in the centre of the beam. They are separated by an almost flat background of $\sim 0.3 \mathrm{~Hz}$ that can be attributed to cosmic particles. A few noisy channels are spotted as isolated peaks over the background, they are however very few and their counting rate is small $(<2.0 \mathrm{~Hz})$.

By applying a cut on the time of the trigger $( \pm 500 \mathrm{~ns})$, cosmics and noise hits are suppressed. This cut removes some hits from beam particles as well. These are interpreted as particles traversing the prototype during a readout. Although vetoed by the trigger, they can still be recorded by the prototype because its dead time is shorter than the one of the telescope and because its electronics is self-triggered.

\subsection{Self-trigger operation}

Thanks to the excellent noise conditions reported in the previous section, the $1 \times 1 \mathrm{~m}^{2}$ prototype can be operated without an external trigger. In this self-trigger mode, no telescope nor trigger electronics are used: the prototype is read out when a memory full signal sent by a MICROROC is received at a DIF board (in trigger mode, a memory full signal resets all chip memories and does not introduce dead time). The beam and voltage settings of the previous test (with external trigger) are used.

A simple way to verify that the prototype is efficient in this mode is to compare the average time between readouts in spill to its expected value. The latter is calculated as the ratio of the memory depth (127 events) to the highest chip counting rate $(\sim 130 \mathrm{~Hz})$ and is roughly $1 \mathrm{~s}$. This is in agreement with measurements as illustrated in Figure 14 (top). Another evidence for an efficient operation in self-trigger mode is shown in Figure 14 (bottom) where the channel counting rates in trigger and self-trigger modes are compared and found similar. Successful operation in self-trigger mode is possible because of the negligible noise rate and discharge rate. Such rates are achieved thanks to a precise electronic calibration and a reliable mesh manufacturing process.

\subsection{Response of the six Micromegas meshes}

The $1 \times 1 \mathrm{~m}^{2}$ prototype was moved across the beam to measure the MIP efficiency and hit multiplicity of the six ASUs. A muon beam of similar intensity as in the previous studies was directed at the centre of each ASU. Roughly $10^{5}$ events were recorded for each position at a mesh voltage of $390 \mathrm{~V}$. Efficiency and hit multiplicity are deduced from the distribution of the number of hits per triggering muons. This distribution is built by finding a track in the telescope, extrapolating its intersection with the prototype and counting the number of hits in time with the trigger inside a search region centred around the pad containing the extrapolated track position. Events are selected by applying the following cuts:

\section{Telescope cut}

Single aligned hits in the three chambers to select tracks with minimum angle w.r.t. the beam axis and to extrapolate the track position at the prototype in the most precise way. This cut reduces the statistics by roughly one third. 


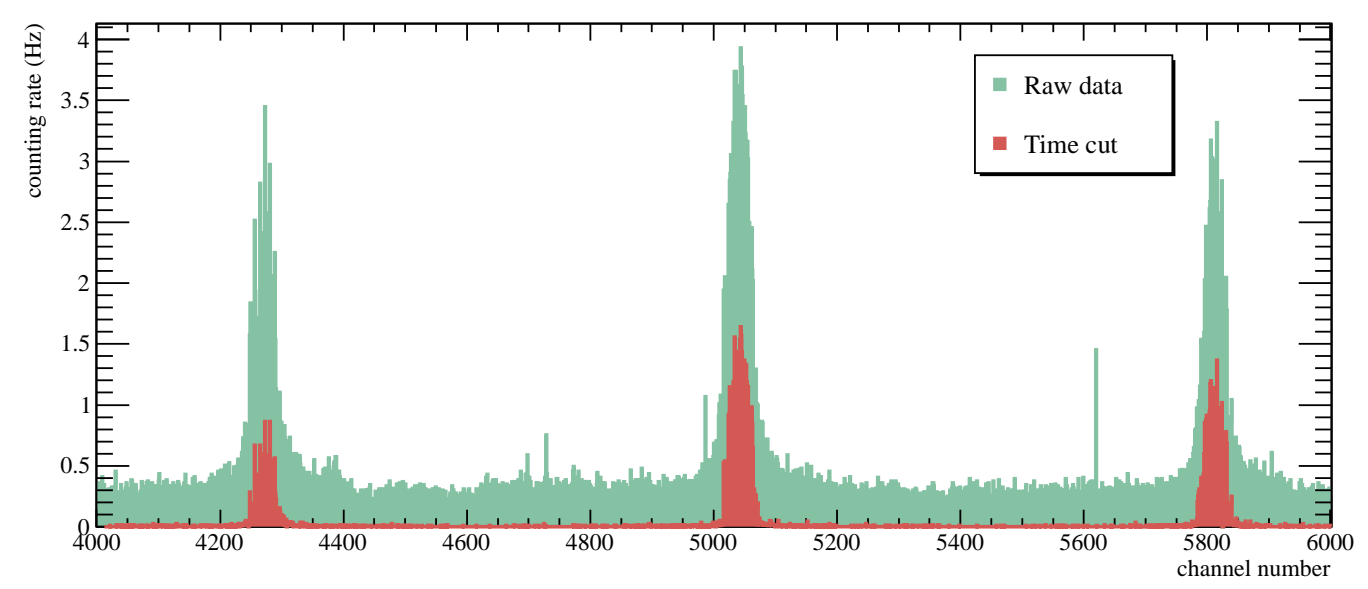

Figure 13: Counting rate of one fifth of the $1 \times 1 \mathrm{~m}^{2}$ prototype channels measured with all hits (Raw data) and for hits in time with the trigger (Time cut). The three broad peaks are interpreted as beam muons, the background between these peaks as cosmic particles and isolated spikes (e.g. channel 5650$)$ as noisy channels.
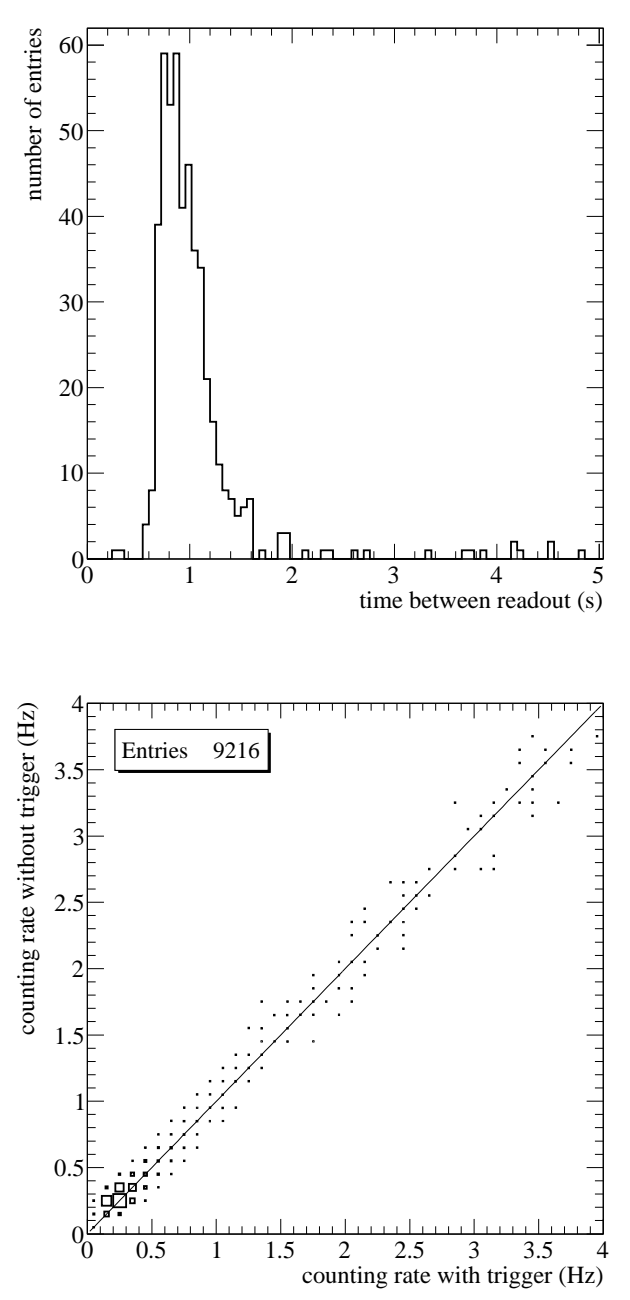

Figure 14: Time between readout in self-trigger mode (top). Channel counting ${ }_{46}$ rate during spills (bottom): self-trigger versus trigger mode.

\section{Prototype cut}

No hits in time with the trigger outside the search region to reduce the impact of multiple scattering on the measured efficiency. The radial distribution of hits (in time) w.r.t. the extrapolated pad falls rapidly and exhibits a long tail from muons scattered in the last telescope chamber. From the distribution shape, a search region of $7 \times 7$ pads is chosen. This cut reduces further the statistics by $5 \%$.

About $30 \times 10^{3}$ events pass the selection for each ASU. They are used to build the distribution of the number of hits above the 3 thresholds. The efficiency $\epsilon$ of a given threshold is calculated as the probability to have at least one hit in the search region:

$$
\epsilon=1-N_{0} / N_{\mathrm{t}}
$$

and the hit multiplicity $m$ as the average number of hits in the search region provided there is at least one hit in the search region:

$$
m=\sum_{\mathrm{i}=1}^{49} i \frac{N_{\mathrm{i}}}{N_{\mathrm{t}}-N_{0}}
$$

where $N_{\mathrm{i}}$ is the number of events with "i" hits and $N_{\mathrm{t}}$ the total number of selected events. Efficiency and hit multiplicity were calculated for the six ASU and for the three thresholds. High efficiency and low hit multiplicity were achieved for the low threshold, with little spread from ASU to ASU (Table 2). Medium and high thresholds were set to 2 and 10 MIP respectively and show smaller values. Because these two thresholds are set within the signal distribution, their response is more sensitive to the detector non-uniformity than the one of the low threshold and indeed, more spread is observed. These variations could be due to small differences of the amplification gap size from one ASU to the other. They are, however, not too large and can be attenuated by adjusting the mesh voltage or the corresponding chip thresholds. In section 4.7 , a way to calculate these corrections using the direct readout of shaper signals is presented. 


\begin{tabular}{c|cccccc} 
ASU & 1 & 2 & 3 & 4 & 5 & 6 \\
\hline \hline$\epsilon_{0}(\%)$ & 97.74 & 97.47 & 98.74 & 98.23 & 98.25 & 96.61 \\
$m_{0}$ & 1.064 & 1.072 & 1.079 & 1.080 & 1.075 & 1.079 \\
\hline$\epsilon_{1}(\%)$ & 34.83 & 36.67 & 46.38 & 40.95 & 38.65 & 46.00 \\
$m_{1}$ & 1.033 & 1.033 & 1.035 & 1.035 & 1.037 & 1.033 \\
\hline$\epsilon_{2}(\%)$ & 3.68 & 3.68 & 4.61 & 3.97 & 4.04 & 4.61 \\
$m_{2}$ & 1.050 & 1.057 & 1.059 & 1.075 & 1.052 & 1.046
\end{tabular}

Table 2: MIP efficiency $\epsilon$ and hit multiplicity $m$ of the six ASU for the three thresholds. The statistical error on $\epsilon$ is below $0.10 \%$ and below 0.008 for $m$.

\subsection{Effect of the peaking time}

The MICROROC chip was designed for various MPGD geometries, for instance with a Bulk mesh of different gap size or with a Gas Electron Multiplier structure [26]. For this purpose, the peaking time of the preamplifier can be set to 75,115 , 150 or $200 \mathrm{~ns}$ (the latter being the default value of the $1 \times 1 \mathrm{~m}^{2}$ prototype). In the gas mixture used, the signal from the multiplication of a single primary electron in a $128 \mu \mathrm{m}$ gap consists of a fast electron peak $(\sim 1 \mathrm{~ns})$ and a longer ion tail $(\sim 100-$ $200 \mathrm{~ns})$. For a traversing MIP, the signal is the sum of, on average, 30 primary electrons arriving at the mesh in about $30 \mathrm{~ns}$ [24]. Therefore, a strong dependence of the efficiency on the peaking time is expected.

This dependence was measured by performing a voltage scan for the four values of the peaking time in the muon beam. The beam was directed to the centre of one ASU and the efficiency calculated as in the previous section. The $150 \mathrm{~ns}$ and $200 \mathrm{~ns}$ trends shown in Figure 15 are similar, meaning that the Micromegas MIP signal is completed in $150 \mathrm{~ns}$ or less. The loss of 500 efficiency from $150 \mathrm{~ns}$ to $115 \mathrm{~ns}$ peaking time indicates, however, that the signal lasts longer than $115 \mathrm{~ns}$ which is compat- ${ }^{507}$ ible with expectations. At shorter peaking times, an efficiency ${ }_{509}^{508}$ larger than $95 \%$ can be maintained by increasing the gas gain. This is illustrated in Table 3 where the voltages for $95 \%$ effi- ${ }^{510}$ ciency are listed: the loss of signal when changing the peaking time from $200 \mathrm{~ns}$ to $75 \mathrm{~ns}$ is compensated by a $20 \mathrm{~V}$ increase of mesh voltage. These voltages are calculated using the empirical ${ }_{514}^{513}$ parametrisation:

$$
\epsilon(V)=\frac{\epsilon_{\max }}{1+\exp \left(\frac{V_{50}-V}{\Delta V}\right)}
$$

where $\epsilon_{\max }$ is the efficiency at infinite voltage, $V_{50}$ is the volt-519 age for $50 \%$ efficiency and $\Delta V$ describes the rise of the $\epsilon(V)_{520}$ trend. All adjusted $\epsilon_{\max }$ parameters are compatible and yield ${ }_{521}$ an average of $99.3 \pm 0.3 \%$. The fact that the $\epsilon_{\max }$ is not equal 522 to one could be explained by the dead ares from the mesh sup-523 porting pillars. The voltage $V_{50}$ decreases at longer peaking ${ }_{524}$ time as a result of the increased available signal and becomes ${ }_{525}$ constant between $115-150 \mathrm{~ns}$. At decreasing peaking times be-526 low $115 \mathrm{~ns}$, the efficiency rise with voltage is steeper which is accounted for by smaller $\Delta V$ values.

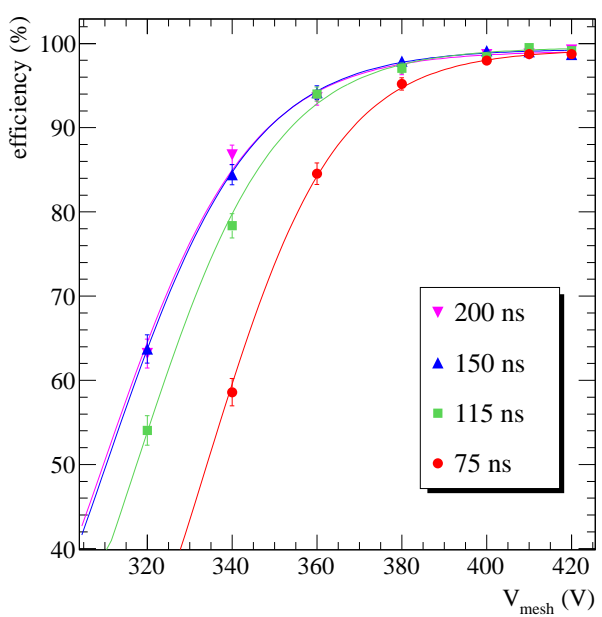

Figure 15: MIP efficiency versus mesh voltage for various settings of the MICROROC peaking time.

\begin{tabular}{c|cccc}
$t_{\mathrm{p}}(\mathrm{ns})$ & 75 & 115 & 150 & 200 \\
\hline \hline$\epsilon_{\max }(\%)$ & $99.3 \pm 0.3$ & $99.6 \pm 0.3$ & $99.4 \pm 0.3$ & $99.1 \pm 0.3$ \\
$V_{50}(\mathrm{~V})$ & $333.9 \pm 0.7$ & $317.4 \pm 0.8$ & $310.1 \pm 0.9$ & $309.4 \pm 0.8$ \\
$\Delta V(\mathrm{~V})$ & $15.2 \pm 0.5$ & $16.3 \pm 0.7$ & $17.0 \pm 0.8$ & $17.1 \pm 0.7$ \\
$V_{95}(\mathrm{~V})$ & 380.9 & 366.7 & 362.6 & 363.0
\end{tabular}

Table 3: Parameters describing the voltage dependence of the efficiency for various settings of the MICROROC peaking time. The voltage $V_{95}$ necessary to reach an efficiency of $95 \%$ is indicated in the last line.

\subsection{Impact of dead zones between ASUs}

Non-instrumented areas inside the prototype amount to $1.5 \%$ of the total area occupied by the six ASUs $\left(96.9 \times 97.4 \mathrm{~cm}^{2}\right)$. Another contribution to the prototype inefficiency may come from possible non-uniformity of the electric field at the ASU edges. This hypothesis was tested by placing a block of iron in a pion beam (collimated to a $3 \times 3 \mathrm{~cm}^{2}$ region) and measuring downstream of the block secondary particles produced in hadron showers. In this way a large fraction of the prototype is exposed and possible discontinuities in the measured hit profile can be looked for. For this measurement, the mesh voltages were set to $375 \mathrm{~V}$.

Given the block size $\left(10 \times 10 \mathrm{~cm}^{2}\right.$ transverse size and $20 \mathrm{~cm}$ length along the beam), roughly half of the pions interacts inside the block. The distribution of the number of hits in the prototype thus shows a peak at one hit from penetrating pions and a long tail up to 300 hits from showering pions. Horizontal and vertical profiles of showers are constructed from events with a hit multiplicity larger than three. They are shown in Figure 16 where a small drop of efficiency for pads at the ASU edges is observed. 

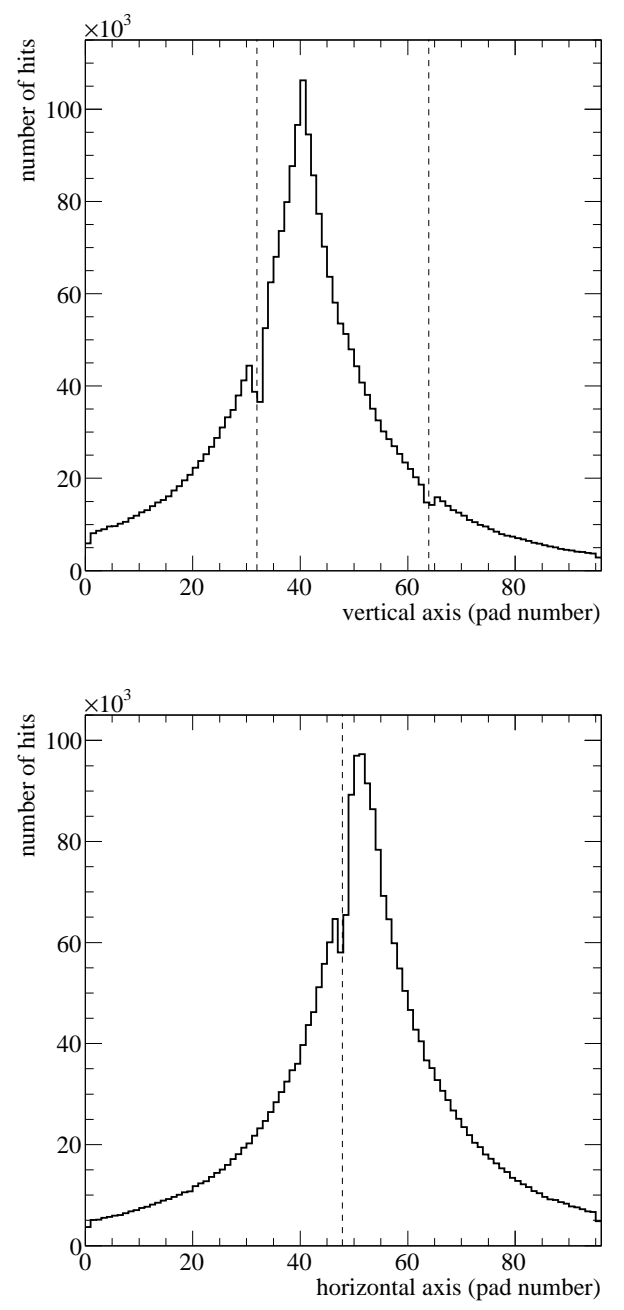

Figure 16: Vertical and horizontal profiles of $150 \mathrm{GeV} / \mathrm{c}$ pion showers $\left(\sim 5 \times 10^{4}\right.$ events). The dashed lines indicate the junctions between ASUs.

By extrapolation of the inner pad occupancy to the ASU546 edges, the number of hits there is $20 \%$ lower than expected. ${ }^{547}$ The number of pads at the ASU edges is 936 which implies ${ }^{548}$ that $10 \%$ of the pads experience a $20 \%$ efficiency loss. The 549 overall resulting inefficiency of $2 \%$ could probably be reduced 550 with different voltage settings (e.g. higher amplification or drift ${ }^{551}$ field) or a different mechanical design (e.g. larger ASUs).

\subsection{Measurement of pion showers with three thresholds}

On average, hadron showers consist of a dense electromag- ${ }^{555}$ netic core from neutral meson decays surrounded by a halo of ${ }^{56}$ particles. Saturation in a DHCAL will be caused mainly by the ${ }^{557}$ electromagnetic part and offline compensation techniques (us- ${ }^{558}$ ing for instance the detector granularity) will be necessary to ${ }^{559}$ improve the energy resolution. In a SDHCAL, the charge infor ${ }^{560}$ mation from the three thresholds can also be exploited to iden ${ }^{-56}$ tify the electromagnetic part. This identification capability can $^{562}$ be illustrated by measuring the threshold efficiencies for various ${ }^{563}$ energy deposits. Because the energy density decreases with the ${ }^{564}$ distance to the shower axis, the efficiencies were measured as $\mathrm{a}^{565}$
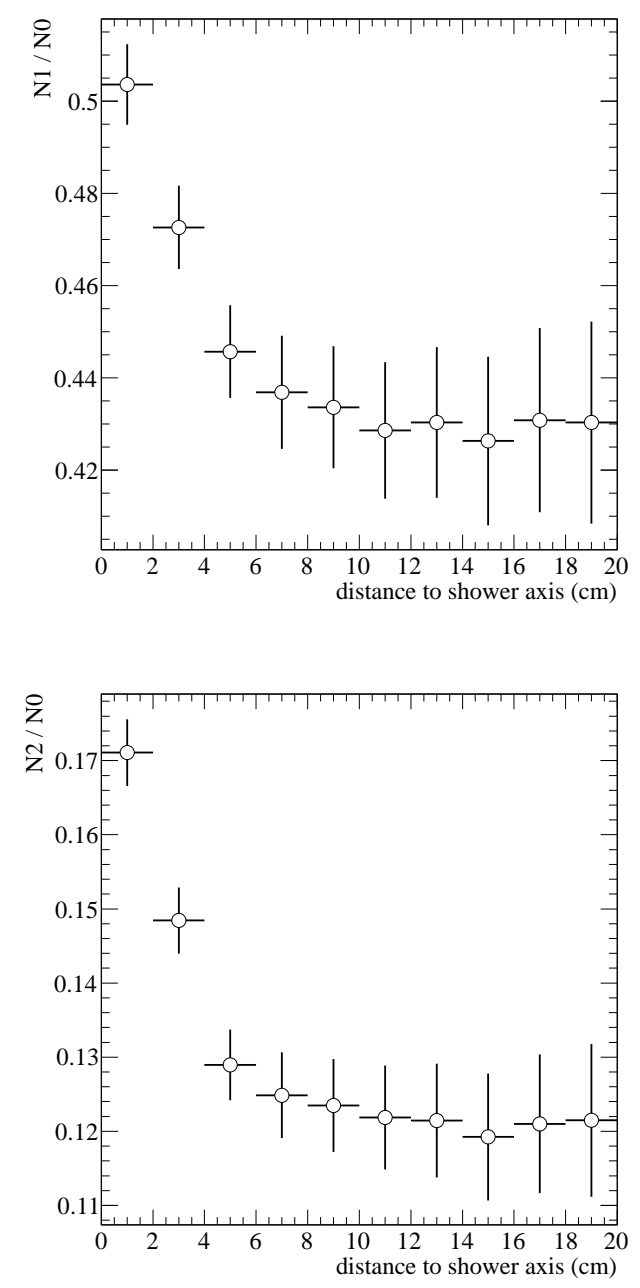

Figure 17: Medium and high threshold efficiency in $150 \mathrm{GeV} / \mathrm{c}$ pion showers versus the distance to the shower axis.

function of position using the set-up described in the previous section. The mesh voltage was $370 \mathrm{~V}$ and the thresholds were set to roughly $(0.5,2,5)$ MIP.

The MIP efficiency of the low threshold is high $(\geq 95 \%)$ and therefore the efficiency of the other thresholds is approximated to $N_{1} / N_{0}$ and $N_{2} / N_{0}$ where the indices 0,1 and 2 stand for low, medium and high thresholds. These ratios are plotted versus distance to the shower axis in Figure 17. Both trends indicate that the electromagnetic core is contained in a circle of $10 \mathrm{~cm}$ radius. This is larger than the $99 \%$ containment radius in iron (3.5 $R_{M} \sim 6 \mathrm{~cm}$ where the Molière radius $R_{M}$ is equal to $1.7 \mathrm{~cm}$ [27]) because of the $1 \mathrm{~m}$ thick air gap between the rear surface of the iron block and the $1 \times 1 \mathrm{~m}^{2}$ prototype.

Compared to the halo of the shower, the core has a higher energy density which explains the probability variation with distance: $N_{1} / N_{0}$ increases from 0.43 to 0.51 and $N_{2} / N_{0}$ from 0.12 to 0.17 . The threshold information can thus help to identify the electromagnetic part of hadron showers and probably improve the calorimeter performance with dedicated software compensation methods. 


\subsection{Analogue readout of the shaper}

A correction of the mesh voltage or of the readout threshold may be necessary to improve the response uniformity of the prototype, in particular for the medium and high thresholds (cf. section 4.3). The most straight-forward way to calculate the correction is to measure the signal distribution. For this reason, dedicated lines were implemented on the PCBs to measure the output voltage of the low gain shaper. This analogue readout uses a trigger signal that first arrives at the DIFs. After a programmable delay matching the peaking time of the MICROROC, the DIFs forward the signal to the chips. The voltages of the shaper outputs of all channels are then multiplexed and sent to the DIFs where they are digitised with a 12 bit resolution.

The analogue readout was tested in the muon beam. The Landau distribution as measured on roughly 100 pads and corrected for channel to channel pedestal variations is shown in Figure 18 (top). By applying cuts on the passed thresholds, the signal distribution is cropped from zero to the threshold value. The latter is thus measured in unit of charge (Figure 18) or in unit of the MIP value which is a natural energy unit in a calorimeter. Threshold and MIP values are determined using the following parametrisation of the charge spectrum:

$$
f(q)=s\left(q, Q_{\mathrm{thr}}, \Delta Q_{\mathrm{thr}}\right) \cdot l\left(q, C, Q_{\mathrm{mpv}}, \Delta Q_{\mathrm{mpv}}\right)
$$

where $s(q)$ is a sigmoid function of inflexion point $Q_{\mathrm{thr}}$, width $\Delta Q_{\text {thr }}$ and with a maximum value of one accounting for the channel to channel threshold dispersion. The function $l(q)$ is the Landau function of most probable value $Q_{\mathrm{mpv}}$, width $\Delta Q_{\mathrm{mpv}}$ and normalisation factor $C$. When adjusting the parameters to the data of Figure 18, it is found that (for this particular run) low, medium and high thresholds are respectively equal to 0.5 , 0.9 and 2.3 times the MIP value of $5.2 \mathrm{fC}$. This shows that the threshold values can be monitored and adjusted during operation using dedicated calibration runs.

\subsection{Power-pulsing of the MICROROC chips}

The MICROROC chip can be turned on and off rapidly according to an external timing signal (e.g. the accelerator clock). When the chip is turned on, an external programmable delay is applied before any detector signal can be recorded to the memory. This delay accounts for the stabilisation of the various voltages and currents inside the chip and should be as short as possible to reduce the power consumption. If the delay is too short, the detector occupancy is dominated by noise until stabilisation. This is illustrated in Figure 19 (left) where the number of hits in the $1 \times 1 \mathrm{~m}^{2}$ prototype is plotted versus time for a short run in self-trigger mode. During the run, the delay was set to $50 \mu$ s and a power-pulsing on/off timing of $3 / 4.5 \mathrm{~s}$ was used. This timing, although different from the one of the ILC bunches $(1 / 199 \mathrm{~ms})$, is well suited to determine the right delay: at $100 \mu \mathrm{s}$, the high peaks every $7.5 \mathrm{~s}$ disappear because stabilisation has been achieved (Figure 19 (right)). With this short stabilisation time, a duty cycle of the front-end electronics at ILC of at most $0.55 \%$ can be achieved, compared to $0.5 \%$ if no stabilisation is needed. The corresponding increase of power consumption would thus be small, below $10 \%$.
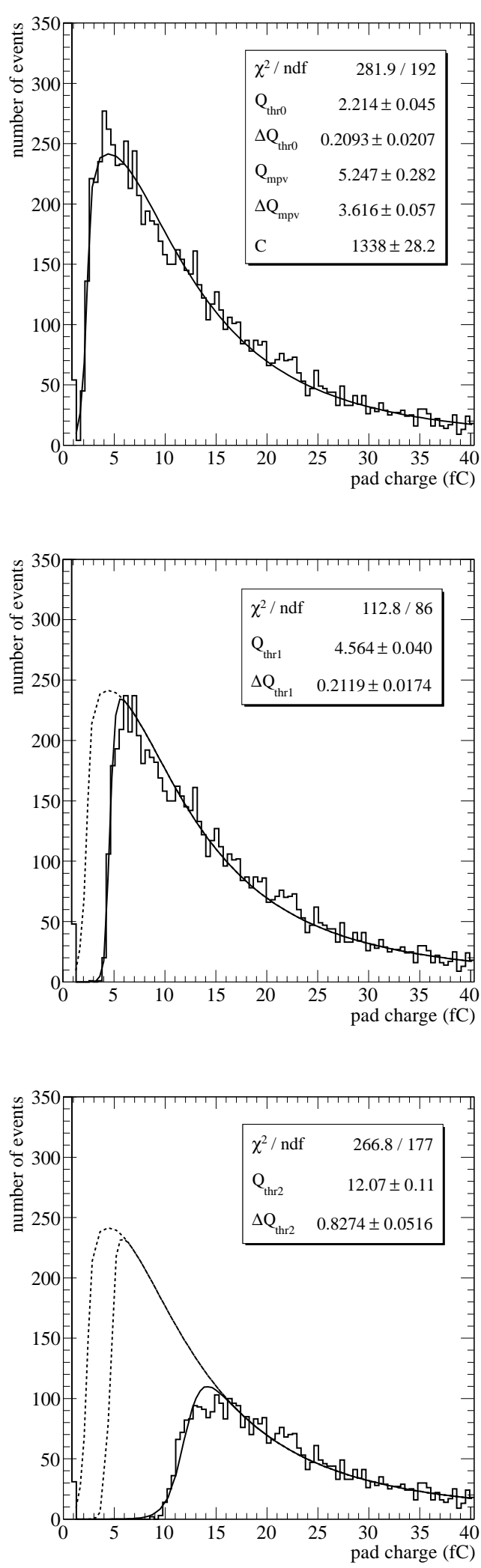

Figure 18: Landau distribution measured with muons for low (top), medium (centre) and high (bottom) threshold hits. 

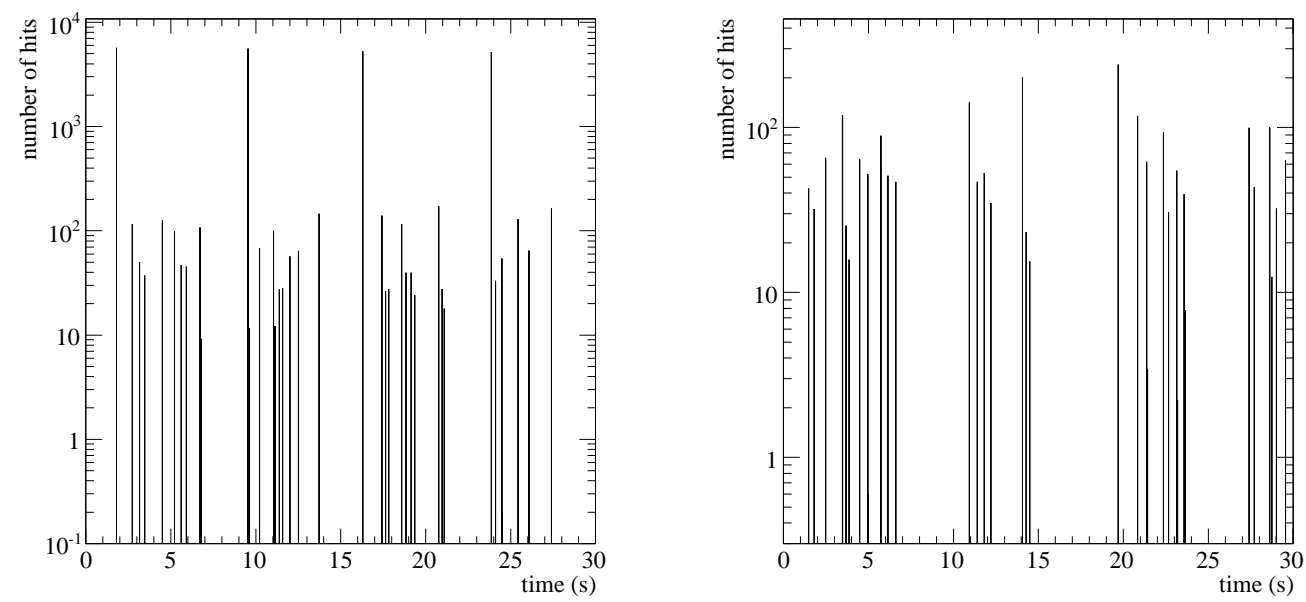

Figure 19: Number of hits versus time when power-pulsing the MICROROC chips of the $1 \times 1 \mathrm{~m}^{2}$ prototype: with $50 \mu \mathrm{s}$ (left) and $100 \mu \mathrm{s}$ (right) delay between the power on signal and the start of the self-trigger acquisition.

\section{Conclusion}

A Micromegas prototype of $1 \times 1 \mathrm{~m}^{2}$ consisting of six in-658 dependent Micromegas boards with integrated 2 bit front-end ${ }^{659}$ electronics has been constructed. This modular design in- ${ }_{661}^{660}$ troduces little dead zones $(\sim 1.5 \%)$ and allows to achieve $a_{662}$ overall thickness of $9.5 \mathrm{~mm}$ and a constant drift gap of $3 \mathrm{~mm} .663$ Thanks to adequate discharge protections and low noise front ${ }^{664}$ end circuits, more than $99.98 \%$ of the 9216 prototype chan- ${ }_{666}^{665}$ nels are operational. Most importantly, the six Micromegas 667 boards exhibit comparable performance to X-rays, muons and ${ }^{668}$ pion showers and all provide the necessary gas gain for an effi- ${ }_{670}^{669}$ ciency of $96 \%$ or larger.

Compared to a pure digital gaseous calorimetry, an approach 672 with three thresholds will rely strongly on the proportionality of 673 the sampling detector and on its cell to cell signal uniformity. ${ }_{675}^{674}$ This kind of Micromegas is free of saturation effects and its $\mathrm{s}_{676}$ amplification gap is precisely defined by the mesh supporting 677 pillars over the anode plane. Small variations of this gap have ${ }^{678}$ probably been observed from mesh to mesh. But the necessary ${ }_{680}^{679}$ corrections to the mesh voltages or the chip thresholds can be ${ }_{681}$ calculated by means of the direct readout of detector signals. ${ }^{682}$ Combined with other features such as power-pulsing and self- ${ }^{683}$ triggering, the constructed Micromegas prototype is therefore ${ }_{685}^{684}$ an excellent candidate for Particle Flow calorimetry at a future ${ }_{686}$ linear collider.

\section{References}

[1] A. Djouadi et al., International Linear Collider Reference Design Report ${ }^{692}$ Volume 2: PHYSICS AT THE ILC, arXiv:hep-ph 0709.1893 (2007).

[2] CLIC Physics Working Group, Physics at the CLIC Multi-TeV Linear ${ }^{694}$ Collider, arXiv:hep-ph 0412251 (2004).

[3] ATLAS collaboration, Observation of a new particle in the search for ${ }^{696}$ the Standard Model Higgs boson with the ATLAS detector at the LHC, ${ }^{697}$ Physics Letters B 716 (2012) 1.

[4] CMS collaboration, Observation of a new boson at a mass of $125 \mathrm{GeV} 699$ with the CMS experiment at the LHC, Physics Letters B 716 (2012) 30. 700

[5] The RD52 experiment, http://highenergy.phys.ttu.edu/dream..
[6] The CALICE collaboration, https://twiki.cern.ch/twiki/bin/view/CALICE.

[7] R. Wigmans, The DREAM project-Results and plans, Nucl. Instr. and Meth. A 572 (2007) 215.

[8] J.-C. Brient, H. Videau, The calorimetry at a future $e^{+} e^{-}$linear collider, arXiv:hep-ex 0202004v1 (2002).

[9] M.A. Thomson, Particle flow calorimetry and the PandoraPFA algorithm, Nucl. Instr. and Meth. A 611 (2009) 25.

[10] V. Andreev et al., A high-granularity scintillator calorimeter readout with silicon photomultipliers, Nucl. Instr. and Meth. A 540 (2005) 368.

[11] B. Bilki et al., Calibration of a digital hadron calorimeter with muons, JINST 3 (2008) 05001.

[12] S. Mannai et al., High granularity Semi-Digital Hadronic Calorimeter using GRPCs, Nucl. Instr. and Meth. A, Article in Press (2012).

[13] A. Ghezzi, T. Tabarelli de Fatis, G. Tinti, Digital Hadron Calorimetry with Glass RPC Active Detectors, arXiv:physics 0507021 (2005).

[14] C. Adloff et al., Monte Carlo study of the physics performance of a digital hadronic calorimeter, JINST 4 (2009) 11009.

[15] SiD Letter of Intent, arXiv/physics.ins-det 0911.0006 (2009).

[16] Y. Giomataris et al., MICROMEGAS: a high-granularity positionsensitive gaseous detector for high particle-flux environments, Nucl. Instr. and Meth. A 376 (1996) 29.

[17] M. Atac et al., Saturated Avalanche Calorimeter, Nucl. Instr. and Meth. 205 (1983) 113

[18] C. Adloff et al., MICROMEGAS chambers for hadronic calorimetry at a future linear collider, JINST 4 (2009) 11023.

[19] I. Giomataris et al., Micromegas in a bulk, Nucl. Instr. and Meth. A 560 (2006) 405

[20] C. Adloff et al., MICROROC: MICRO-mesh gaseous structure Read-Out Chip, JINST 7 (2012) 01029.

[21] Omega, microelectronics for physics and medical imaging: http://omega.in2p3.fr/.

[22] M. Chefdeville, Development of Micromegas-like gaseous detectors using a pixel readout chip as collecting anode, CERN Document Server PhD Thesis (2009) 151.

[23] S.F. Biagi, Monte Carlo simulation of electron drift and diffusion in counting gases under the influence of electric and magnetic fields, Nucl. Instr. and Meth. A 421 (1999) 234.

[24] F. Sauli, Principles of operation of Multiwire Proportional and Drift Chambers, CERN Yellow Reports 77-09 (1977).

[25] J. Bouchez et al., Bulk Micromegas detectors for large TPC applications, Nucl. Instr. and Meth. A 547 (2007) 425.

[26] F. Sauli, GEM: A new concept for electron amplification in gas detectors, Nucl. Instr. and Meth. A 386 (1997) 531.

[27] K. Nakamura et al., Review of Particle Physics, Journal of Physics G 37 (2010) 070521. 